\title{
SECULAR TRENDS IN MATERNAL PREPREGNANCY BMI AND GESTATIONAL WEIGHT GAIN OVER A 15 YEAR PERIOD IN GREECE
}

\author{
G. Giannouli ${ }^{1}$, V. Bakoulas ${ }^{2}$, A. Veltsista ${ }^{1}$, A. Palili ${ }^{1}$, V. Lekea ${ }^{1}$, C. Bakoula ${ }^{1}$ \\ ${ }^{1} 1$ st Department of Paediatrics, University of Athens, Aghia Sophia Children's Hospital, ${ }^{2} 2$ nd Department of \\ Obstetrics and Gynecology, University of Athens, Aretaieio Hospital, Athens, Greece
}

Background and aims: Secular trends in maternal prepregnancy BMI and gestational weight gain (GWG) by BMI class [based on the 2009 Institute of Medicine (IOM) guidelines], and association with birthweight, adjusted for maternal age, parity, smoking and education, from 1983 to 1998 in Greece were investigated.

Methods: Data was derived from two cross-sectional population surveys. In total, 7027 and 11275 women aged 18-40 years with a singleton full-term live birth were assessed in 1983 and 1998, respectively. Categories below and above the 2009 IOM recommendations as a reference were created.

Results: The mean age and prepregnancy BMI of all pregnant mothers increased between 1983 and 1998 (from 26.2 to 28.5 years, from 21.5 to $22.2 \mathrm{~kg} / \mathrm{m} 2$ ). A rise was observed in both the overweight and obese BMI categories (from 12 to $15.3 \%$ and from 2.1 to $4.7 \%$ ). However, the proportion of women with less than appropriate GWG increased significantly in the 1998 (37.8\% vs. 33.0\%), compared to the 1983 cohort. In both cohorts, less than appropriate GWG by BMI class was significantly associated with small-forgestational-age (SGA), adjusted for mother's age, education, parity and smoking, and attenuated the association between maternal prepregnancy BMI and birthweight.

Conclusions: Despite the rising rates of overweight and obese women, there was an increase in the proportion of those with less than appropriate GWG. It could be that mothers' attitude towards weight gain has changed over time. Of importance, weight gain during pregnancy has to be monitored tightly as low GWG is associated with SGA infants. 